PROCEEDINGS OF THE

AMERICAN MATHEMATICAL SOCIETY

Volume 136, Number 10, October 2008, Pages 3405-3408

S 0002-9939(08)09557-9

Article electronically published on May 29, 2008

\title{
A HOPF ALGEBRA HAVING A SEPARABLE GALOIS EXTENSION IS FINITE DIMENSIONAL
}

\author{
JUAN CUADRA \\ (Communicated by Birge Huisgen-Zimmermann) \\ To José Luis Gómez Pardo on the occasion of his 60th birthday
}

\begin{abstract}
It is shown that a Hopf algebra $H$ over a field admitting a Galois extension $A$ separable over its subalgebra of coinvariants $B$ is of finite dimension. This answers in the affirmative a question posed by Beattie et al. in [Proc. Amer. Math. Soc. 128, No. 11 (2000), 3201-3203]. It is also proven that this result holds true if $H$ has bijective antipode and the extension $A / B$ is Frobenius.
\end{abstract}

\section{INTRODUCTION}

The notion of Hopf-Galois extension, as known nowadays, is due to Kreimer and Takeuchi [7] and it is a mainstay of Hopf algebra theory. It originated in the work of Chase and Sweedler about actions of Hopf algebras on rings 3. When the Hopf algebra is the coordinate algebra of an affine group scheme that acts on an affine algebra, the above notion may be interpreted in geometric terms and it is linked with the concept of torsor or principal homogeneous space [10, page 168]. Faithfully flat Hopf-Galois extensions are currently widely accepted as a noncommutative counterpart of this geometric concept.

For $H=k[G]$, the group algebra of a group $G$ over a field $k$, [8, Theorem 8.1.7] shows that an $H$-Galois extension is precisely a strongly graded algebra, that is, a $k$-algebra $A$ admitting a decomposition $A=\bigoplus_{\sigma \in G} A_{\sigma}$ as $k$-vector space and satisfying $A_{\sigma} A_{\tau}=A_{\sigma \tau}$ for all $\sigma, \tau \in G$. The subalgebra of coinvariants of $A$ is $A_{e}$ (e the identity element of $G$ ). Năstăsescu et al. characterized in [9, Proposition 2.1] when the extension $A / A_{e}$ is separable. In particular, they found that if $A$ is separable over $A_{e}$, then $G$ is finite. It was investigated in 1 if an analogous result could hold for general Hopf algebras. To be more precise, suppose that $H$ is a Hopf algebra over $k$ having a Hopf-Galois extension separable over its subalgebra of coinvariants. Is $H$ necessarily finite dimensional? A positive answer was given under the additional assumption that $H$ is co-Frobenius.

In this short note we answer this question in the affirmative. Our proof relies on a combination of the properties of the separability idempotent, the Galois maps

Received by the editors January 17, 2007, and, in revised form, March 1, 2007, and March 13, 2007.

2000 Mathematics Subject Classification. Primary 16W30.

This research was supported by projects MTM2005-03227 from MCYT and FEDER and P06FQM-1889 from Junta de Andalucía.

(c)2008 American Mathematical Society Reverts to public domain 28 years from publication 
and an old result of Sweedler. As often happens in Hopf algebra theory, the new proof seems more natural and simpler than the original proof for $H=k[G]$. Combining our result with one of Cohen and Fischman we provide a characterization of separable Hopf-Galois extensions that generalizes to Hopf algebras the abovementioned one of Năstăsescu et al. for strongly graded rings. We finally show that if a Hopf algebra $H$ with bijective antipode admits a Hopf-Galois extension $A$ with subalgebra of coinvariants $B$ such that $A / B$ is Frobenius, then $H$ is also finite dimensional.

We fix some notation and recall the definition of Hopf-Galois extension. We expect that the reader is familiar with the rudiments of Hopf algebra theory. Our conventions and notation are those of 8 . Throughout $H$ stands for a Hopf algebra over a field $k$. All vector spaces considered in the sequel are over $k$, map means linear map, and $\otimes$ denotes the tensor product over $k$.

For a right $H$-comodule algebra $A$ with structure map $\rho: A \rightarrow A \otimes H$, its subalgebra of coinvariants $A^{c o(H)}=\left\{a \in A: \rho(a)=a \otimes 1_{H}\right\}$ is denoted by $B$. The canonical Galois maps are given by

$$
\begin{aligned}
\text { can }: A \otimes_{B} A \rightarrow A \otimes H, & a \otimes_{B} a^{\prime} \mapsto \sum_{\left(a^{\prime}\right)} a a_{(0)}^{\prime} \otimes a_{(1)}^{\prime}, \\
c a n^{\prime}: A \otimes_{B} A \rightarrow A \otimes H, & a \otimes_{B} a^{\prime} \mapsto \sum_{(a)} a_{(0)} a^{\prime} \otimes a_{(1)} .
\end{aligned}
$$

Recall from [8, Definition 8.1.1] that $A / B$ is said to be an $H$-Galois extension if can is bijective. If $H$ has bijective antipode, then can is surjective or bijective if and only if $c a n^{\prime}$ is [8, page 124].

\section{The MAIN THEOREM}

Theorem. Let $H$ be a Hopf algebra and $A$ a right $H$-comodule algebra with subalgebra of coinvariants $B$. Suppose that can : $A \otimes_{B} A \rightarrow A \otimes H$ is surjective and there is a nonzero element $e=\sum_{i=1}^{n} e_{i} \otimes_{B} e_{i}^{\prime} \in A \otimes_{B} A$ such that ae $=$ ea for all $a \in A$ and $\operatorname{can}^{\prime}(e) \neq 0$. Then $H$ is finite dimensional.

Proof. We will prove that $H$ has a nonzero finite dimensional left ideal. In virtue of Sweedler's result [11, Corollary 2.7] (see alternatively [5, Lemma 5.3.1(i)]), this will imply that $H$ is finite dimensional.

We pick nonzero elements $a_{j} \in A, h_{j} \in H$ for $j=1, \ldots, m$ such that

$$
\sum_{j=1}^{m} a_{j} \otimes h_{j}=\operatorname{can}^{\prime}(e)=\sum_{i=1}^{n} \sum_{\left(e_{i}\right)} e_{i(0)} e_{i}^{\prime} \otimes e_{i(1)}
$$

and the $a_{j}$ 's are linearly independent. Take $h \in H$ arbitrary. Since can is surjective we may find $c_{l}, d_{l} \in A$ for $l=1, \ldots, r$ satisfying

$$
1 \otimes h=\operatorname{can}\left(\sum_{l=1}^{r} c_{l} \otimes_{B} d_{l}\right)=\sum_{l=1}^{r} \sum_{\left(d_{l}\right)} c_{l} d_{l(0)} \otimes d_{l(1)} .
$$

For $l=1, \ldots, r$ we have $d_{l} e=e d_{l}$. Applying $c a n^{\prime}$ to this equality we get

$$
\sum_{i=1}^{n} \sum_{\left(e_{i}\right)} \sum_{\left(d_{l}\right)} d_{l(0)} e_{i(0)} e_{i}^{\prime} \otimes d_{l(1)} e_{i(1)}=\sum_{i=1}^{n} \sum_{\left(e_{i}\right)} e_{i(0)} e_{i}^{\prime} d_{l} \otimes e_{i(1)} .
$$


Then,

$$
\begin{aligned}
& \sum_{j=1}^{m} a_{j} \otimes h h_{j} \stackrel{10(2)}{=} \sum_{l=1}^{r} \sum_{\left(d_{l}\right)} \sum_{i=1}^{n} \sum_{\left(e_{i}\right)} c_{l} d_{l(0)} e_{i(0)} e_{i}^{\prime} \otimes d_{l(1)} e_{i(1)} \\
& \stackrel{\underline{\underline{3}}}{=} \quad \sum_{l=1}^{r} \sum_{i=1}^{n} \sum_{\left(e_{i}\right)} c_{l} e_{i(0)} e_{i}^{\prime} d_{l} \otimes e_{i(1)} \\
& \stackrel{11}{=} \quad \sum_{l=1}^{r} \sum_{j=1}^{m} c_{l} a_{j} d_{l} \otimes h_{j} \text {. }
\end{aligned}
$$

Let $\varphi_{t} \in A^{*}$ be such that $\varphi_{t}\left(a_{j}\right)=\delta_{t j}$, the Kronecker symbol, for $t=1, \ldots, m$. Evaluating $\varphi_{t} \otimes i d_{H}$ on the preceding set of equalities we obtain

$$
h h_{t}=\sum_{l=1}^{r} \sum_{j=1}^{m} \varphi_{t}\left(c_{l} a_{j} d_{l}\right) h_{j} .
$$

This yields that the subspace spanned by the $h_{j}$ 's is a finite dimensional nonzero left ideal of $H$, as required.

Assume that $A / B$ is separable and let $e=\sum_{i=1}^{n} e_{i} \otimes_{B} e_{i}^{\prime} \in A \otimes_{B} A$ be the separability idempotent. Then $\sum_{i=1}^{n} e_{i} e_{i}^{\prime}=1_{A}$ and $a e=e a$ for all $a \in A$. Notice that $\operatorname{can}^{\prime}(e)$ is nonzero since

$$
1_{A}=\sum_{i=1}^{n} e_{i} e_{i}^{\prime}=\sum_{i=1}^{n} \sum_{\left(e_{i}\right)} e_{i(0)} \varepsilon\left(e_{i(1)}\right) e_{i}^{\prime}=\left(i d_{A} \otimes \varepsilon\right) \operatorname{can}^{\prime}(e),
$$

where $\varepsilon$ denotes as usual the counit of $H$. Applying the above theorem we get the announced result.

Corollary 1. Let $H$ be a Hopf algebra and $A$ a right $H$-Galois extension separable over its subalgebra of coinvariants $B$. Then $H$ is finite dimensional.

Cohen and Fischman provided in [4, Theorem 1.8] several characterizations of separable Hopf-Galois extensions for a finite dimensional Hopf algebra. These characterizations together with our result allow us to characterize such extensions for an arbitrary Hopf algebra.

Corollary 2. Let $H$ be a Hopf algebra and $A$ a right $H$-Galois extension. Then, $A / A^{c o(H)}$ is separable if and only if $H$ is finite dimensional and one of the equivalent conditions (2)-(6) in [4, Theorem 1.8] holds.

This corollary may be viewed as a generalization to Hopf algebras of 9 , Proposition 2.1] characterizing strongly graded rings separable over the degree one component. Under the assumption that $H$ has bijective antipode, Corollary 1 holds true for Frobenius extensions. We would like to thank the referee for pointing out this fact.

Corollary 3. Let $H$ be a Hopf algebra having bijective antipode and $A$ a right $H$ Galois extension with subalgebra of coinvariants B. If $A / B$ is Frobenius, then $H$ is finite dimensional.

Proof. The hypothesis on $A / B$ assures the existence of a nonzero element $e \in$ $A \otimes_{B} A$ such that $a e=e a$ for all $a \in A$, [6, Remarks 1.2(a) and 1.4(c)]. Since $H$ has bijective antipode, $c a n^{\prime}$ is bijective. Our theorem now applies. 
When $B$ is a field and $A$ a $B$-algebra, $A / B$ separable implies $A / B$ is finite dimensional and semisimple, which in turn implies $A / B$ is Frobenius [2, Section 1.3].

\section{REFERENCES}

[1] M. Beattie, S. Dăscălescu, and Ş. Raianu, A Co-Frobenius Hopf Algebra with a Separable Galois Extension Is Finite. Proc. Amer. Math. Soc. 128, No. 11 (2000), 3201-3203. MR.1690974 (2001b:16040)

[2] S. Caenepeel, G. Militaru, and S. Zhu, Frobenius and Separable Functors for Generalized Module Categories and Nonlinear Equations. Lecture Notes in Mathematics 1787, SpringerVerlag, Berlin, 2002. MR1926102 (2003h:16061)

[3] S.U. Chase and M.E. Sweedler, Hopf Algebras and Galois Theory. Lecture Notes in Mathematics 97, Springer-Verlag, Berlin, 1969. MR0260724 (41:5348)

[4] M. Cohen and D. Fischman, Semisimple Extensions and Elements of Trace 1. J. Algebra 149 (1992), 419-437. MR.1172438 (93c:16038)

[5] S. Dăscălescu, C. Năstăsescu, and Ş. Raianu, Hopf Algebras. An Introduction. Monographs and Textbooks in Pure and Applied Mathematics 235, Marcel-Dekker, New York, 2001. MR.1786197 (2001j:16056)

[6] D. Fischman, S. Montgomery, and H.-J. Schneider, Frobenius Extensions of Subalgebras of Hopf Algebras. Trans. Amer. Math. Soc. 349, No. 12 (1997), 4857-4895. MR1401518 (98c:16049)

[7] H.F. Kreimer and M. Takeuchi, Hopf Algebras and Galois Extensions of an Algebra. Indiana Univ. Math. J. 30 (1981), 675-692. MR625597 (83h:16015)

[8] S. Montgomery, Hopf Algebras and Their Actions on Rings. CBMS Regional Conf. Ser. in Math. 82, AMS, 1993. MR1243637 (94i:16019)

[9] C. Năstăsescu, M. Van den Bergh, and F. Van Oystaeyen, Separable Functors Applied to Graded Rings. J. Algebra 123 (1989), 397-413. MR.1000494 (90j:16001)

[10] H.-J. Schneider, Principal Homogeneous Spaces for Arbitrary Hopf Algebras. Israel J. Math. 72, Nos. 1-2 (1980), 167-195. MR1098988 (92a:16047)

[11] M.E. Sweedler, Integrals for Hopf Algebras. Ann. of Math. (2) 89 (1969), 323-335. MR0242840 (39:4167)

Universidad de Almería, Depto. Álgebra y AnÁlisis Matemático, E-04120 Almería, SPAIN

E-mail address: jcdiaz@ual.es 